\title{
A comparison of diceCT and histology for determination of nasal epithelial type
}

\author{
Timothy D Smith ${ }^{\text {Corresp., } 1}$, Hayley M Corbin ${ }^{2}$, Scot King ${ }^{1}$, Kunwar P Bhatnagar $^{3}$, Valerie B DeLeon ${ }^{4}$ \\ ${ }^{1}$ School of Physical Therapy, Slippery Rock University, Slippery Rock, PA, USA \\ 2 Department of Biology, Slippery Rock University, Slippery Rock University, Slippery Rock, PA, United States \\ 3 Department of Anatomical Sciences and Neurobiology, University of Louisville, Louisville, KY, USA \\ 4 Department of Anthropology, University of Florida, Gainesville, Florida, United States \\ Corresponding Author: Timothy D Smith \\ Email address: timothy.smith@sru.edu
}

Diffusible iodine-based contrast-enhanced computed tomography (diceCT) has emerged as a viable tool for discriminating soft tissues in serial CT slices, which can then be used for three-dimensional analysis. This technique has some potential to supplant histology as a tool for identification of body tissues. Here, we studied the head of an adult fruit bat (Cynopterus sphinx) and a late fetal vampire bat (Desmodus rotundus) using diceCT and $\mu \mathrm{CT}$. Subsequently, we decalcified, serially sectioned and stained the same heads. The two CT volumes were rotated so that the sectional plane of the slice series closely matched that of histological sections, yielding the ideal opportunity to relate CT observations to corresponding histology. Olfactory epithelium is typically thicker, on average, than respiratory epithelium in both bats. Thus, one investigator (SK), blind to the histological sections, examined the diceCT slice series for both bats and annotated changes in thickness of epithelium on the first ethmoturbinal (ET I), the roof of the nasal fossa, and the nasal septum. A second trial was conducted with an added criterion: radioopacity of the lamina propria as an indicator of Bowman's glands. Then, a second investigator (TS) annotated images of matching histological sections based on microscopic observation of epithelial type, and transferred these annotations to matching CT slices. Measurements of slices annotated according to changes in epithelial thickness alone closely track measurements of slices based on histologically-informed annotations; matching histological sections confirm blind annotations were effective based on epithelial thickness alone, except for a patch of unusually thick non-OE, mistaken for $\mathrm{OE}$ in one of the specimens. When characteristics of the lamina propria were added in the second trial, the blind annotations excluded the thick non-OE. Moreover, in the fetal bat the use of evidence for Bowman's glands improved detection of olfactory mucosa, perhaps because the epithelium itself was thin enough at its margins to escape detection. We conclude that 
diceCT can by itself be highly effective in identifying distribution of $\mathrm{OE}$, especially where observations are confirmed by histology from at least one specimen of the species. Our findings also establish that iodine staining, followed by stain removal, does not interfere with subsequent histological staining of the same specimen. 
1 A comparison of diceCT and histology for determination of nasal epithelial type

2 Timothy D. Smith, ${ }^{1}$ Hayley M. Corbin, ${ }^{2}$ Scot E.E. King, ${ }^{1}$ Kunwar P. Bhatnagar, ${ }^{3}$ Valerie B.

3 DeLeon $^{4}$

4

5 1, School of Physical Therapy, Slippery Rock University, Slippery Rock, PA, USA

6 2, Department of Biology, Slippery Rock University, Slippery Rock, PA, USA

7 3, Department of Anatomical Sciences and Neurobiology, University of Louisville, Louisville

8 KY, USA

9 4, Department of Anthropology, University of Florida, Gainesville, FLA, USA

11 Corresponding author:

12 Tim D. Smith, Ph.D.

13 School of Physical Therapy

14108 Central Loop

15 Slippery Rock University

16 Slippery Rock, PA 16057, USA

17 Email: timothy.smith@sru.edu

18

19

20 


\begin{abstract}
Diffusible iodine-based contrast-enhanced computed tomography (diceCT) has emerged as a viable tool for discriminating soft tissues in serial CT slices, which can then be used for threedimensional analysis. This technique has some potential to supplant histology as a tool for identification of body tissues. Here, we studied the head of an adult fruit bat (Cynopterus sphinx) and a late fetal vampire bat (Desmodus rotundus) using diceCT and $\mu \mathrm{CT}$. Subsequently, we decalcified, serially sectioned and stained the same heads. The two CT volumes were rotated so that the sectional plane of the slice series closely matched that of histological sections, yielding the ideal opportunity to relate CT observations to corresponding histology. Olfactory epithelium is typically thicker, on average, than respiratory epithelium in both bats. Thus, one investigator (SK), blind to the histological sections, examined the diceCT slice series for both bats and annotated changes in thickness of epithelium on the first ethmoturbinal (ET I), the roof of the nasal fossa, and the nasal septum. A second trial was conducted with an added criterion: radioopacity of the lamina propria as an indicator of Bowman's glands. Then, a second investigator (TS) annotated images of matching histological sections based on microscopic observation of epithelial type, and transferred these annotations to matching CT slices. Measurements of slices annotated according to changes in epithelial thickness alone closely track measurements of slices based on histologically-informed annotations; matching histological sections confirm blind annotations were effective based on epithelial thickness alone, except for a patch of unusually thick non-OE, mistaken for OE in one of the specimens. When characteristics of the lamina propria were added in the second trial, the blind annotations excluded the thick non-OE. Moreover, in the fetal bat the use of evidence for Bowman's glands improved detection of olfactory mucosa, perhaps because the epithelium itself was thin enough at its margins to escape detection. We conclude that diceCT can by itself be highly effective in identifying distribution of $\mathrm{OE}$, especially where observations are confirmed by histology from at least one specimen of the species. Our findings also establish that iodine staining, followed by stain removal, does not interfere with subsequent histological staining of the same specimen.
\end{abstract}

Key Words: imaging; mammals; mucosa; nasal cavity; olfactory neuroepithelium 


\section{Introduction}

Diffusible iodine-based contrast-enhanced computed tomography (diceCT) has emerged as a viable tool for discriminating soft tissues in serial CT slices, which can then be used for three-dimensional analysis. This has already been used to study multiple soft tissues, including muscle (Cox \& Jeffery, 2011; Gignac et al., 2016; Orsbon, Gidnark, \& Ross, 2018; Santana, 2018; Dickenson et al., 2019), nervous (Girard et al., 2016), epithelial (Gignac \& Kley, 2014; Yohe, Hoffmann \& Curtis, 2018) and other tissues. The arrival of this technique holds much promise to replace the other traditional means of studying soft tissue structures, such as microdissection and histology, both of which are destructive techniques that permanently alter specimens (DeLeon \& Smith, 2016; Hedrick et al., 2018). However, for many purposes even high resolution computed tomography currently lacks the ability to match histology in its capacity to identify extremely small anatomical structures (e.g., Reinholt et al., 2009). In the present study we explore the capacity of diceCT for detecting internal nasal tissues. If diceCT can suffice for histology to some extent, the technique may have the major advantage to markedly decrease the laborious time involved in quantification or three-dimensional reconstructions using histology (e.g., Smith et al., 2007; Maier and Ruf, 2014; Yee et al., 2016), while also providing increased sample sizes.

There are four commonly described types of epithelium that line the nasal cavity, of which two predominate (Harkema et al., 2006; Smith and Bhatnagar, 2019). There are relatively small amounts of stratified epithelia that mainly line drainage routes and the vestibule, and a type of poorly known function called transitional epithelium. The vast majority of the nasal cavity is lined with respiratory and olfactory epithelia. In most mammals, respiratory epithelia is the predominant type anteriorly and inferiorly within the nasal cavities, and is recognizable based on pseudostratified, columnar structure, the presence of unicellular glands (goblet cells), and apical cilia that are observable by light microscopy (Harkema et al., 2006; Smith and Bhatnagar, 2019). Olfactory epithelium (OE) is typically the predominant type posterodorsally; it is also pseudostratified, but has more numerous rows of nuclei throughout its thickness compared to respiratory epithelium. Most rows of nuclei are those of olfactory sensory neurons. Cilia are also present at the epithelial apex of OE, but they are enmeshed within a mucous covering that typically obscures them when viewed by light microscopy (Dennis et al., 2015; Smith et al., 2019). OE is generally thicker than non-olfactory types of epithelium (Smith et al., 2021). Using diceCT, Yohe and colleagues observed thickened epithelia along ethmoturbinals and other turbinals that bear most of the OE (Yohe, Hoffmann \& Curtis, 2018). Tahara \& Larsson (2013), using diceCT to study quail visceral tissues, suggested both cellular density and cytoplasmic storage may promote radioopacity of epithelial tissues. Since OE is typically thicker than nonolfactory types (Weiler \& Farbman, 1997), this suggests diceCT may be used in lieu of histology for identifying internal nasal tissues. However, Yohe, Hoffmann \& Curtis (2018) also observed that transition points between olfactory and respiratory epithelia are not detectable using diceCT alone. Nonetheless, these authors did observe some characteristics of the underlying lamina 
propria that helped to identify respiratory mucosa (specifically, glandular masses). This raises an important issue regarding olfactory tissues. In olfactory mucosa, there are glands present in the underlying connective tissue (or, lamina propria), intermingled with olfactory nerve bundles. Called Bowman's glands, these branched tubular masses are often densely packed (Smith \& Bhatnagar, 2019). Based on basic characteristics of respiratory and olfactory mucosa, the glandular masses in the latter might be detected based on their uniform opacity, as contrasted to the more isolated "islands" of radiopaque masses that signify respiratory glands (Yohe, Hoffmann \& Curtis, 2018).

Here, we studied the head of an adult fruit bat (Cynopterus sphinx) and a late fetal vampire bat (Desmodus rotundus) using high resolution diceCT. We seek to identify epithelial and mucosal (i.e., epithelium + lamina propria) transition points using diceCT, with an added reference of histology subsequent to CT scanning. This method of examining individual specimens using both techniques was recently used to great advantage by Girard et al. (2016) to study murine brain lesions. In our methodological study, we assess the potential of diceCT to supplant histology as a tool for identification of olfactory mucosa.

\section{Materials and Methods}

Two bat species were selected, including two different ages. For a relatively large bat, an adult Cynopterus sphinx was included in our study. A far smaller specimen, a late fetal Desmodus rotundus, was also selected to determine limitations that may relate to size of the specimen. Both specimens are part of an archival collection of preserved and histologically sectioned bats in the collection of KPB, now curated by TDS. The Desmodus specimens in this collection, including a pregnant female with a late fetus, were originally collected in Veracruz, Mexico, in the 1980s (Bhatnagar, 2008). The Cynopterus specimen was collected in Jhabua, India (Cooper and Bhatnagar, 1976). Both specimens were originally fixed in $10 \%$ buffered formalin. The Desmodus fetus was fixed still within its amnionic sac (its mother was dissected open through the abdominal wall and uterine wall to enhance fixation). The Cynopterus was a full head and partial cervical region. Subsequent to fixation, both specimens were transferred to $80 \%$ ethanol and stored in the decades since, with periodic changes of fluid. Thus, the two specimens provided a parallel to common museum practice in terms of storage, and also two different stages of maturation and head size. Use of these specimens for the study was approved by the Institutional Animal Care and Use Committee at Slippery Rock University (IACUC protocol \# 2021-03T).

Each specimen was scanned using traditional $\mu-\mathrm{CT}$ and diceCT methods. Subsequently, each head was bisected and then serially sectioned in the coronal plane and stained with two procedures, hematoxylin-eosin and Gomori trichrome. 
Conventional $\mu-\mathrm{CT}$ scanning was conducted for the fetal Desmodus specimen at Northeast Ohio Medical University (NEOMED) using a Scanco vivaCT 75 scanner (scan parameters: $70 \mathrm{kVp} ; 114 \mathrm{~mA}$ ) and reconstructed with $0.0205 \times 0.0205 \times 0.0205 \mathrm{~mm}$ cubic voxels. Conventional $\mu$-CT scans of the adult Cynopterus specimen were collected at the University of Florida with a GE V|tome|xm 240 CT scanner (scan parameters: $100 \mathrm{kVp} ; 100$ $\mathrm{mA}$ ) and reconstructed with $0.0213 \times 0.0213 \times 0.0213 \mathrm{~mm}$ cubic voxels. Specimens were prepared for diceCT at the University of Florida following the protocols outlined in Gignac et al., 2016. Briefly, specimens were submerged in 20\% sucrose solution for 24-48 hours, and subsequently submerged in Lugol's iodine $\left(\mathrm{I}_{2} \mathrm{KI}\right)$. The adult Cynopterus specimen was placed in a 5\% Logol's solution for seven days. The fetal Desmodus specimen was originally placed in a 5\% Lugol's solution for 34 days, but this resulted in overstaining of the specimen and poor imaging outcomes. The specimen was destained by submerging in a 5\% sodium thiosulfate $\left(\mathrm{Na}_{2} \mathrm{~S}_{2} \mathrm{O}_{3}\right)$ solution. More recently we have optimized our diceCT protocol to include lower concentrations of Lugol's iodine (e.g., 1\%) over longer periods of time, refreshing the solution periodically. The fetal Desmodus specimen was again submerged in 20\% sucrose solution for 48 hours, and subsequently submerged in 1\% Lugol's iodine for seven days. DiceCT images were collected at the University of Florida GE V|tome|xm 240 CT scanner. The fetal Desmodus specimen was scanned using parameters of $160 \mathrm{kVp}$ and $100 \mathrm{~mA}$ with a $0.5 \mathrm{~mm}$ copper filter and reconstructed with $0.0181 \times 0.0181 \times 0.0181 \mathrm{~mm}$ cubic voxels. The adult Cynopterus specimen was scanned using parameters of $100 \mathrm{kVp}$ and $140 \mathrm{~mA}$ (no filter) and reconstructed with 0.0256 x 0.0256 x $0.0256 \mathrm{~mm}$ cubic voxels. Specimens were subsequently destained by submerging in a $5 \%$ sodium thiosulfate $\left(\mathrm{Na}_{2} \mathrm{~S}_{2} \mathrm{O}_{3}\right)$ solution.

Histological sectioning was completed at the neurohistology laboratory in the School of Physical Therapy, Slippery Rock University. Each specimen was decalcified in a formic acidsodium citrate solution with weekly tests to detect completion. After decalcification, the specimens were paraffin embedded, serially sectioned at $10 \mu \mathrm{m}$, and every $4^{\text {th }}$ to $5^{\text {th }}$ section was mounted and stained. All histological observations were conducted by TDS, using a Leica DMLB photomicroscope at X200 to X630.

A major step preceding cross-referencing histology and CT is the alignment of crosssectional planes (Fig. 1). To do this, we identified corresponding features in the CT and histological data, and used these to reconstruct the plane of section in the CT volume. The CT volume was then digitally re-sliced using Amira 2019 software, such that orthogonal sections of the CT image corresponded to histological sections. In this study, all alignment was optimized for the caudal half of the nasal fossa, which contains most of the ethmoturbinal complex. A more detailed account of these methods is presented in DeLeon \& Smith (2014), and all alignments were conducted by VBD. Prior to observing diceCT slices, aligned diceCT slices were modified using the "auto contrast" function, via an automated batch command, using Adobe Photoshop software. This heightened the contrast of epithelium and lamina propria in the Cynopterus scan, but not appreciably so in the Desmodus scan. 
In the first analysis, descriptive characteristics of nasal epithelia were assessed in the adult Cynopterus to add to existing data in the literature. All nasal tissues appeared exceptionally well-preserved. The observer (TS) identified olfactory mucosa according to the presence of rows of olfactory sensory neuronal bodies as is typical of OE, and the presence of Bowman's glands and olfactory nerves in the underlying lamina propria (Harkema, Carey \& Pestka 2006; Smith \& Bhatnagar, 2019). Adjacent non-OE bore kinocilia, and was thus respiratory epithelium of varying morphology. Based on these characteristics, using the adult Cynopterus, thickness of OE was measured in ImageJ using X200 micrographs of seven sites (see Fig. S1), including: a) dorsal rim of ethmoturbinal I, b) lateral margin of nasal septum near its intersection with dorsal apex of the nasal chamber, c) ventral rim of ethmoturbinal I, converging with ventral rim of ethmoturbinal II, d) lateral margin of nasal septum, near its intersection with the palate, e) medial margin of frontoturbinal 2, f) dorsal rim of nasoturbinal, and g) "roof" or dorsal apex of nasal fossa. Selected other sites were sampled for measuring to demonstrates range of thickness on non-OE, such as non-OE patches on the nasoturbinal, frontoturbinal, ethmoturbinal III, and a thick patch of non-OE found rostrally. For epithelial measurements, the sites in Fig. S1 and selected other sites were photographed in multiple sections (at every $16^{\text {th }}$ to $32^{\text {nd }}$ section, totaling 15 to 24 measurements per site) in which the structure was present. A photograph of a stage micrometer at the same x200 magnification was used to set the scale in ImageJ. The height of the epithelium in each section was measured using a line tool drawn from the basal to apical sides of the epithelium, with the line oriented at a right angle to the basement membrane. A single factor analysis of variance (ANOVA) was used to assess whether significant $(p<0.05)$ differences exist in olfactory epithelial thickness among the five OE sites shown in Fig. S1 (a, b, e, f, g). In addition, we used t-tests comparing thickness (in $\mu \mathrm{m}$ ) of olfactory epithelium (OE) versus nonolfactory epithelium (non-OE) on ethmoturbinal I (sites a vs. c) and the nasal septum (sites b vs. d). It should be noted that here the word "turbinal" is most frequently used to denote a mucosalined bony structure, rather than the bone itself, which would bear the same name.

The second analysis tested artifactual changes as a result of processing for diceCT and histology in the adult Cynopterus specimen. Multiple studies have observed artifactual changes to tissues with histological or diceCT methods. The dehydration steps that preceded paraffin embedding are known to produce extreme shrinkage artefacts manifested in stained sections (Tahara \& Larson, 2013; DeLeon \& Smith, 2014). As noted above, shrinkage artifacts are also manifested following diceCT processing, but Tahara \& Larson (2013) assert diceCT-related shrinkage is similar to that resulting from fixation. Here, we expected shrinkage of tissues in both specimens due to the original treatment with a high concentration of Lugol's solution (i.e., $5 \%$ ). We sought to confirm this by measuring perimeters of selected structures visible in the $\mu \mathrm{CT}$ slices obtained using traditional scans, and then comparing these to the same measurements in matching diceCT slides, and to the histology sections to which both were aligned. Two sites were chosen for this analysis based on their isolation from other tissues: the maxilloturbinal and an epiturbinal (Figs. 2a,d). Paired t-tests were conducted to compare measurements of matching slice levels to assess for significant $(\mathrm{p}<0.05)$ differences. 
204

205

206

207

208

209

210

211

212

213

214

215

216

217

218

219

220

221

222

223

224

225

226

227

228

229

230

231

232

233

234

235

236

237

238

239

The third analysis tested the ability to discriminate $\mathrm{OE}$ and non-OE from diceCT images. The perimeter of the OE was measured on diceCT slices using ImageJ software. One observer (TS) annotated the histology-aligned diceCT slices by directly comparing them to matching histological sections. The histological section was viewed through on a monitor linked to a Axiocam MRc 5 Firewire camera attached to Leica DMLB compound microscope. Simultaneously, the matching diceCT slice was viewed on a second computer monitor using ImageJ software, and annotated according to the limits of olfactory epithelium on selected structures as determined by microscopic examination; the paint tool in ImageJ was used to annotate limits of olfactory epithelium based on matching contours (Fig. S2C). Two structures were selected for annotation of OE in both bat specimens: the combined septum/roof of the nasal fossa and the first ethmoturbinal (Fig. S2). These were annotated on each diceCT slice from the attachment site of the first ethmoturbinal as a caudal limit, and rostrally to the rostral limit of the olfactory mucosa. This region was selected because previous work on other mammals showed that transitions in epithelial type occur on both of these structures (Smith et al., 2007; Pang et al., 2016). Because histological measurements confirmed significant differences in OE versus non-OE thickness, as reported in other mammals (Weiler \& Farbman, 1997), a second observer (SK) blindly annotated transitions from OE to non-OE based on changes in epithelial thickness in diceCT slices (Fig. S3), focusing on the region matching the histology series.

Subsequently, a second blind trial of the third analysis was performed which considered tissues deep to the epithelium. Mucous membranes, or mucosae, have two components that relate to its functional characteristics: the surface epithelium and the underlying, supportive lamina propria. Thus, a second trial was conducted blindly by SK, using an added criterion: characteristics of the lamina propria. Yohe, Hoffmann \& Curtis (2018) observed that radiopaque glands may be indicative of respiratory mucosa. By viewing matched histological sections and CT slices, we observed this is also true of Bowman's glands. Thus, in a second trial, SK blindly annotated olfactory mucosa in diceCT slices based on the combined criteria of relatively thick epithelium and relatively higher radioopacity of the lamina propria deep to it. Because Kolmogorov-Smirnov tests revealed half of the data were not normally distributed, we transformed all the data $\left(\log _{10}\right)$ prior to analysis. The measurements were compared to the blindly annotated perimeters in matching series of sections using a repeated measures two-way (ANOVA) testing the effects of location and annotation type in SPSS software. Post hoc testing for between-groups differences was done using Fisher's Least Significant Differences (LSD) test.

\section{RESULTS}

Alignment of CT and histology 
240

241

242

243

244

245

246

247

248

249

250

251

252

253

254

255

256

257

258

259

260

261

262

263

264

265

266

267

268

269

270

271

272

273

274

Alignment of $\mu \mathrm{CT}$ and diceCT volumes to the plane of histology resulted in an excellent correspondence of structures throughout the head in the fetal Desmodus. Some shrinkage of mucosa in histology made the airways appear larger in cross-section (Fig. 1a), but contours matched well (Figs. 1a-c). Alignment of $\mu \mathrm{CT}$ and diceCT volumes to the plane of histology in the adult Cynopterus resulted in excellent correspondence of structures in some regions, but rostrally the matching of contours was less precise. This was particularly so with respect to free projections of turbinals, which are known to shrink more than attached structural elements (DeLeon and Smith, 2014). However, very precise contour matching was accomplished in the more caudal olfactory region (Figs. 1d-f). All CT scan slices are available on MorphoSource at the project link (https://www.morphosource.org/projects/000365326). The examination of diceCT and histology in matching planes, from the same specimens, provided an ideal opportunity to confirm tissue identity based in microanatomical characteristics (see below).

\section{Analysis 1: Epithelial metrics in adult Cynopterus based on histology}

In the adult Cynopterus, most non-OE of the nasal cavity (excluding the vestibule) is ciliated columnar or ciliated pseudostratified columnar in morphology, with a broad range of thickness, from 6.3 to $51.7 \mu \mathrm{m}$. However, the thickest patch of non-OE was restricted to a zone just rostral to the first ethmoturbinal, and also rostral to the most rostral appearance of olfactory mucosa (as verified using histology). Aside from this patch, the thickest non-OE was $21.2 \mu \mathrm{m}$. Olfactory mucosa ranged from 26.3 to $71.6 \mu \mathrm{m}$ in thickness based on a sampling of multiple turbinals and other surfaces. Among five locations of OE measured, a one-way single-factor ANOVA reveals significant $(\mathrm{p}<0.001)$ differences based on site of measurement (Table 1). More specifically, LSD tests reveal epithelia of ET I and nasoturbinal are significantly thinner compared to all other olfactory sites, but not significantly different from each other (Table 1). One apparent trend in Cynopterus is that the free margins of turbinal projections have relatively thinner OE, whereas measurements taken from the septum, the roof of the nasal fossa, and along planar surfaces of turbinals are thicker.

To assess whether thickness of epithelial types on individual structures is distinctive, we compared selected histological measurement sites on the first ethmoturbinal and on the nasal septum (sites "a" versus "c" and "b" versus "d" from Fig. S1). Independent t-tests, assuming unequal variance, indicate $\mathrm{OE}$ is significantly thicker on both structures (Table 2). 
275

276

277

278

279

280

281

282

283

284

285

286

287

288

289

290

291

292

293

294

295

296

297

298

299

300

301

302

303

304

305

306

307

308

309

310

311

Two structures that were distinctly visible in $\mu$-CT scan slices (Figs. 2a, d; maxilloturbinal and epiturbinal) were measured and then remeasured in matching, aligned diceCT slices (Figs. 2b, e) and histological sections (Figs. 2c, f) of the adult Cynopterus. We expected that tissue shrinkage due to processing would yield perimeters that are greater in measurements taken from $\mu$-CT slices compared to diceCT or histology.

$\mu-\mathrm{CT}-$ based measurements of the maxilloturbinal are an average of $0.13 \mathrm{~mm}$ greater $(\sim$ $5 \%$ difference) in perimeter compared to the same measurement in diceCT slices (Table S1). $\mu$ CT-based measurements of the epiturbinal, an accessory flange of ET I, are an average of 0.11 mm greater ( $\sim 3 \%$ difference) in perimeter compared to the same measurement in diceCT slices (Table S2). A paired t-test reveals that measurements of the maxilloturbinal from $\mu$-CT slices are significantly greater than those from matching diceCT slices $(t=11.1 ; p<0.001)$. Similarly, a paired t-test reveals that measurements of the epiturbinal from $\mu$-CT slices are significantly greater than those from matching diceCT slices $(t=18.59, \mathrm{p}<0.0001)$. When matching $\mu-C T$ and diceCT slice levels plotted against one another, measurements are nearly parallel (Fig. $2 \mathrm{~g}$, $\mathrm{h}$ ), and $\mu$-CT slice measurements are mostly but not always greater than those from diceCT. The parallel nature of measurements, when plotted against matching slice levels, suggests the slices are well-aligned and that the difference is consistent.

The number of paired comparisons of measurements from histology relative to matching $\mu$-CT slices were fewer, since not all sections were used for staining. However, comparisons of data from matching sections suggest an even greater disparity between measurements based on $\mu$-CT versus histology at matching levels. $\mu$-CT-based-measurements of the maxilloturbinal are an average of $0.33 \mathrm{~mm}$ greater ( $\sim 14 \%$ difference) in perimeter compared to the same measurement in matching histological sections (Table S1). $\mu$-CT-based measurements of the epiturbinal, an accessory flange of ET I, are an average of $0.33 \mathrm{~mm}$ greater $(\sim 11 \%$ difference $)$ in perimeter compared to the same measurement in histology slices (Table S2). A paired t-test reveals that measurements of the maxilloturbinal from $\mu$-CT slices are significantly greater than those from matching histology sections $(t=22.27, \mathrm{p}<0.0001)$. A paired $\mathrm{t}$-test reveals that measurements of the epiturbinal from $\mu$-CT slices are also significantly greater than those from matching diceCT slices $(\mathrm{t}=13.24, \mathrm{p}<0.0001)$.

\section{Criteria for identifying olfactory mucosa using diceCT}

Rough qualitative comparisons of epithelial thickness are possible using diceCT, and sometimes reveal the approximate limits of OE (e.g., Figs. S2c, d). However, since epithelial thickness sometimes falls close to the CT voxel dimensions, at least based on histology (Tables 1-2), we expected that the diceCT images in this study might lack the resolution to establish epithelium type based on epithelial thickness alone. Nonetheless, our diceCT-histology matches indicate that $\mathrm{OE}$ may be qualitatively identified by its thickness and high degree of radioopacity 
312

313

314

315

316

317

318

319

320

321

322

323

324

325

326

327

328

329

330

331

332

333

334

335

336

337

338

339

340

341

342

343

344

345

346

347

compared to non-OE (Figs. 2e, 3c). The degree of radioopacity may relate to density of nuclei of sensory neurons (Fig. 3). Thus, thickness and radioopacity were two criteria used in our attempt to identify the boundaries between non-olfactory and olfactory epithelia.

Most olfactory portions of ethmoturbinals have relatively thick $\mathrm{OE}$ and underlying connective tissue (lamina propria). In both the fetal and adult bat, thickness of olfactory mucosa (epithelium and lamina propria) is greatest on the medial side of ethmoturbinals (Figs. 3a, d). These parts of the turbinals have epithelia that exhibit staggered locations of olfactory sensory neurons throughout epithelial depth, as evidenced by the rows of nuclei (Fig. 3b), and the lamina propria is packed with Bowman's glands and olfactory nerves (Figs. 3b, e). A comparison to corresponding, aligned diceCT slices reveals that the olfactory mucosa lining these parts of the ethmoturbinals are highly radiopaque (Figs. 3c, f), especially by comparison to more ventrally positioned structures such as the maxilloturbinal (Figs. 2b, e). Such lamina propria can appear uniformly opaque in diceCT (e.g., Fig. 3f) or may have a "mottled" appearance with radioopaque patches just deep to mucosal surface; these patches are visible even where the epithelium is indistinct (Fig. 3f, inset).

Just as thickness of olfactory epithelium varies (Tables 1-2), so does thickness of the lamina propria deep to it. For example, histology confirms convex sides of turbinals have a thicker, more densely glandular lamina propria than concave (meatal) surfaces (Fig. d).

Nevertheless, in a matching diceCT slices, the lamina propria is radioopaque on both sides (Figs. 3f). Some small patches of non-OE that interrupt the continuity of OE may be difficult to detect. While easily identifiable using histology (Fig. 3d, black arrow), they are less distinct in diceCT (Fig. 3f, white arrow).

\section{Analysis 3: Perimeter of olfactory surfaces in the region of the rostral part of ethmoturbinal I}

\section{Trial 1: Assessing thickness changes in epithelial thickness using diceCT}

Blind annotations of epithelial changes from OE to non-OE in diceCT (by coauthor SK, blind to histology) were mostly successful in the case of the adult Cynopterus. For the most part, measurements of OE perimeter in slices annotated according to changes in epithelial thickness alone closely track measurements of slices based on histologically-informed annotations (coauthor TS, based on histology) (Fig. S3). However, for both the ethmoturbinal and the septum/roof, "blind" annotations of diceCT images overestimated the amount of OE at the rostral end. This suggests thicker non-OE exists rostrally, which was verified by examination of histology in this region (Fig. S2i). Thus, the majority of data points are parallel between diceCT and histological annotated series, but rostrally the perimeters diverge (see right side of plot in Fig. S2e). 
In the adult Cynopterus, repeated measures two-way ANOVA reveals significant differences between perimeters measured based on blind annotations versus histology-informed annotations of diceCT slices based on location $(\mathrm{F}=9.193 ; \mathrm{p}<0.01)$, but not annotation type $(\mathrm{F}=$ $1.205 ; \mathrm{p}>0.05)$, or interaction $(\mathrm{F}=1.27 ; \mathrm{p}>0.05)$. Perimeters of $\mathrm{OE}$ in the roof/septum measured by the two methods differ by $0.52 \mathrm{~mm}$ on average, with slices annotated blindly measuring less. This difference accounts for $26 \%$ of the average OE perimeter measured from histology-annotated slices. Blind annotations of the ethmoturbinal yield OE perimeter measurements that are $0.16 \mathrm{~mm}$ less, on average, than slices annotated according to histology (Table S3). This difference accounts for $10 \%$ of the average OE perimeter measured from histology-annotated slices.

In the fetal Desmodus, repeated measures two-way ANOVA reveals significant differences between perimeters measured based on blind annotations using diceCT versus histology of structures based on location $(\mathrm{F}=12.667 ; \mathrm{p}<0.01)$, and based on type of annotation $(F=49.864 ; p<0.001)$, but no significant interaction effect $(F=0.106 ; p>0.05)$. Blind annotations of the roof/septum yielded OE perimeter measurements that were $0.62 \mathrm{~mm}$ less, on average, than diceCT slices annotated with reference to histology (Table S4). This difference accounts for $17 \%$ of the average OE perimeter measured from histology-annotated diceCT slices. Blind annotations of the ethmoturbinal yielded OE perimeter measurements that were $0.47 \mathrm{~mm}$ greater, on average, than diceCT slices annotated according to histology (Table S4). This accounts for $22 \%$ of the average OE perimeter measured from histology-annotated slices.

\section{Trial 2: Assessing thickness changes in mucosa using diceCT}

When the epithelium and lamina propria are considered together, side-by-side comparison of perimeters measured from diceCT slices compared to matching histological sections reveal a closer match. Most of the radiopaque, thick mucosa on diceCT corresponds to olfactory mucosa as verified using histology (Fig. 3). The perimeter of OE on the first ethmoturbinal and the nasal roof/septum was annotated in a second trial using the combined criteria of epithelial thickness and degree of radioopacity of the lamina propria.

In the adult Cynopterus, repeated measures two-way ANOVA reveals significant differences between perimeters measured based on blind annotations using diceCT versus histology of structures based on location $(F=30.4 ; p<0.01)$, annotation type $(F=19.13$; $p<$ $0.01)$, and a significant interaction $(\mathrm{F}=5.002 ; \mathrm{p}<0.05)$. In both the ethmoturbinal and the septum/roof, perimeters blindly annotated for olfactory mucosa limits closely parallel measurements informed by histology (Fig. 4). Blind annotations of the ethmoturbinal yield olfactory mucosa perimeter measurements that were $0.04 \mathrm{~mm}$ greater, on average, than slices annotated according to histology (Table S5). This accounts for $2 \%$ of the average olfactory mucosa perimeter measured from histology-annotated slices. Blind annotations of the 
385

386

387

388

389

390

391

392

393

394

395

396

397

398

399

400

401

402

403

404

405

406

407

408

409

410

411

412

413

414

415

416

417

418

419

420

roof/septum yield perimeter measurements that were $0.24 \mathrm{~mm}$ greater, on average, than slices annotated according to histology (Table S5). This difference accounts for $10 \%$ of the average perimeter measured from histology-annotated slices.

In the fetal Desmodus, repeated measures two-way ANOVA reveals significant differences between perimeters measured based on blind annotations using diceCT versus histology of structures based on location $(\mathrm{F}=17.112 ; \mathrm{p}<0.01)$, but no significant difference based on type of annotation $(F=0.762 ; p>0.05)$ or interaction effect $(F=0.119 ; p>0.05)$. Blind annotations of the roof/septum yield olfactory mucosa perimeter measurements that were 0.06 $\mathrm{mm}$ greater, on average, than slices annotated according to histology (Table S6). This difference accounts for $2 \%$ of the average perimeters measured from histology-annotated slices. Blind annotations of the first ethmoturbinal yield perimeter measurements that were $0.09 \mathrm{~mm}$ greater, on average, than slices annotated according to histology (Table S6). This accounts for $2 \%$ of the average OE perimeter measured from histology-annotated slices. Nevertheless, for both sites, comparing measurements by slice shows that "blind" annotations of olfactory mucosa closely parallel measurements informed by histology (Fig. 4).

\section{DISCUSSION}

The increased availability of high resolution $\mu \mathrm{CT}$ has offered a valuable means of studying minute anatomical structures. Perhaps the greatest benefit has been the ability to nondestructively study rare specimens (Hedrick et al., 2018; Yapuncich et al., 2019); this is particularly of value for the study of endangered species or valuable museum samples (Smith et al., 2020). Recently, the availability of diceCT has provided an innovative means to virtually dissect soft-tissue structures, such as individual muscles (e.g., Santana, 2018; Dickenson et al., 2019, 2020) or visceral structures (e.g., Vickerton, Jarvis \&, Jeffery, 2013). Still, $\mu$ CT has not achieved the resolution necessary to match histology in efforts to establish the finest osteological features, such as microscopic bony bridging across sutures at early points of fusion, while still maintaining overall spatial context (Reinholt et al., 2009). Similarly, although diceCT discriminates muscles and organs based on anatomical context, it does not allow discrimination of specific connective epithelial tissues (Yohe, Hoffmann \& Curtis, 2018). For these aims, histology remains the gold standard.

However, in the same way that gross anatomical spatial relationships allow the identification of specific muscles in diceCT studies (Santana, 2018; Dickenson et al., 2019), microanatomical elements of body tissues also provide critical context to infer tissue types. Here, we show that the tissue composition of the lamina propria varies, and the thickness of the nasal epithelia varies, in such a way to make identification of olfactory mucosa possible using diceCT. 
421

422

423

424

425

426

427

428

429

430

431

432

433

434

435

436

437

438

439

440

441

442

443

444

445

446

447

448

449

450

451

452

453

454

455

456

457

458

\section{Artefactual changes to specimens relating to diceCT and histological processing methods}

DiceCT has the potential to resolve several drawbacks to the use of histology to study vertebrate microanatomy. Most notably, the iodine immersion involved in diceCT is reversible, at least to a great extent (Girard et al., 2016), whereas histology permanently limits the use of tissues for study using most other methods, aside from bright-field microscopy and three dimensional reconstruction; i.e., it is a highly destructive technique that is not ideal for examination of rare specimens (DeLeon \& Smith, 2014; Hedrick et al., 2018). Even though histological sections present unparalleled microscopic detail, the cross-sectional plane is permanent, whereas computed tomography data may be manipulated for multiplanar examination (DeLeon \& Smith, 2014). Moreover, histological processing introduces artefactual changes, such as tissue shrinkage and folding (Rolls \& Farmer, 2008). When used for threedimensional reconstructions, this leads to quantifiable distortions (e.g., reduced length dimensions) of structures (DeLeon \& Smith, 2014). While this can be corrected (Smith et al., 2019), diceCT remains a potential alternative. However, diceCT also introduces artifacts such as tissue shrinkage (Hedrick et al., 2018), as demonstrated by the results here.

Before discussing the extent of shrinkage to which diceCT or histology may induce on nasal structures, we should acknowledge uncertainty regarding the extent of shrinkage caused by fixation and long-term ethanol storage. Multiple studies have documented that ethanol storage, especially long-term storage, causes marked shrinkage in soft tissue structures (e.g., Hedrick et al., 2018; Leonard et al., 2021). Some structures appear more susceptible than others (e.g., eyes), but it is also demonstrable that intact, whole animal specimens shrink less than isolated organs or tissue blocks (Fox et al., 1985). We might infer that undecalcified bone of whole specimens is the most important tissue that resists shrinkage, since bone as a tissue shrinks far less than soft tissue organs during histological processing that involves dehydration (Buytaert et al., 2014). And we also suspect that immature specimens with less fully calcified bones shrink more than adult samples. With the uncertainty regarding the extent of shrinkage in mind, the samples used in the present study are very similar to museum samples in that they have been stored in ethanol for decades. In that respect, the results inform us as to the potential value of diceCT for studying museum fluid collections.

Here, we provide quantitative confirmation that both diceCT and histology result in tissue shrinkage of nasal tissues, as is known for other regions/structures (e.g., Hedrick et al., 2018). We draw this inference based on a comparison to $\mu$-CT slices, which can fortuitously allow examination of soft tissue contours within the nasal cavity. Recently, Smith et al. (2021) were able to examine mucosal surfaces in a cadaveric dog snout in high resolution $\mu$-CT scan slices, and even measure mucosal thickness, demonstrating some utility of $\mu$-CT for soft tissue studies, though epithelia were not observable. Several mucosal structures are visible in our samples. Most mucosal contours were obscured, likely due to the presence of fluid, which we assume may be more apt to remain in the snouts of small mammals. However, two structures (the

Peer] reviewing PDF | (2021:05:61578:1:2:NEW 17 Aug 2021) 
459

460

461

462

463

464

465

466

467

468

469

470

471

472

473

474

475

476

477

478

479

480

481

482

483

484

485

486

487

488

489

490

491

492

493

494

495

496

maxilloturbinal and an epiturbinal) fortuitously had exposed contours, enabling a comparison of these structures across corresponding $\mu-\mathrm{CT}$, diceCT, and histology sections.

Shrinkage artifacts are a well-known artifactual change associated with the diceCT procedure (Tahara \& Larsson, 2013; Vickerton, Jarvis \&, Jeffery, 2013). Both high concentration of iodine solutions (Vickerton, Jarvis \&, Jeffery, 2013) and greater durations of immersion for staining (Gignac et al., 2016) may cause more extreme shrinkage. Based on our experience, we currently prefer to use lower concentrations of Lugol's solution (usually 1\%) for specimens of this size, and we perform test scans (as possible) to ensure adequate staining and avoid extended, unnecessary immersion which may result in further shrinkage of the tissue. In addition, some authors have observed differential shrinkage among different tissue types, such as the brain and eyes (Vickerton, Jarvis \&, Jeffery, 2013; Hedrick et al., 2018). Staining isolated tissues samples also causes more extreme shrinkage (Vickerton, Jarvis \&, Jeffery, 2013), while iodine staining of whole specimens is known to produce far less dramatic reductions (Tahara \& Larsson, 2013; Hedrick et al., 2018). Our results confirm that diceCT is associated with reductions in epithelial perimeters as well, by approximately 3 to $5 \%$. This is similar to a $5 \%$ reduction in embryonic quail cranial length following iodine staining (Tahara and Larsson, 2013).

The 11-14 \% differences between $\mu$-CT-based and histology-based perimeters most likely reflects additional shrinkage of the tissue during graded ethanol baths prior to paraffin embedding (Tahara \& Larsson, 2013). This large artefactual distortion means previous quantitative studies of epithelia, at least those based on paraffin-embedding of fixed decalcified tissues (e.g., Adams, 1972; Bhatnagar \& Kallen, 1975; Gross et al., 1982; Smith and Rossie, 2008), likely report distortions of epithelial surface areas of nasal fossa structures. These may be underestimations for external perimeters (e.g., the epiturbinal and maxilloturbinal described here), or overestimations for internal perimeters (e.g., the roof/septum described here). It may be notable that turbinals are supported by especially thin bone, and such structures may shrink to a greater extent than other surfaces with more substantial support, such as the peripheral contours of the nasal fossa (e.g., septum). Indeed, in one recent study we corrected for shrinkage of the rostral projection of the first ethmoturbinal (DeLeon \& Smith, 2014).

While no method other than scans of fresh tissues can be expected to eliminate shrinkage, both diceCT and histology provide a powerful means of tissue differentiation. If epithelial measurements using diceCT can match or approach the accuracy of histology for epithelial tissue identification, then it would have a great advantage of far less shrinkage artifacts when used for the study of whole specimens. Thus, our findings demonstrate the great potential of diceCT for studying rare, valuable specimens (e.g., museum samples) nondestructively, and with less distortions than is seem using histology (DeLeon and Smith, 2014). In addition, we demonstrate that iodine staining followed by stain removal using sodium thiosulfate does not interfere with histological study of well-preserved specimens using traditional techniques such as trichrome staining. 
498

499

500

501

502

503

504

505

506

507

508

509

510

511

512

513

514

515

516

517

518

519

520

521

522

523

524

525

526

\section{7}

528

529

530

531

532

\section{Identification of epithelia using histology and diceCT}

In both bat species, OE is easily identifiable based on well-established light microscopic characteristics such as the presence of rows of cell bodies of olfactory sensory neurons and elongated cilia (e.g., Chamanza \& Wright, 2015; Dennis et al., 2015). The latter are not individually observable by light microscopy, but do stain as a narrow band whereas tangled cilia exist within a mucous film. In contrast, the shorter kinocilia of respiratory epithelium are identifiable at higher magnification.

Another feature of $\mathrm{OE}$ is its greater relative thickness, on average, compared to most nonolfactory types (Dieulafe, 1906). This relates to the distinctive lamina propria that supports it, in which Bowman's glands and bundles of olfactory axons are nested (Chamanza \& Wright, 2015; Dennis et al., 2015). However, in the adult Cynopterus there is a wide range of OE thickness (26.3 to $71.6 \mu \mathrm{m}$ ) and significant differences among sampled structures in the mean OE thickness (Table 2). This result agrees with findings on large samples of postnatal rats, in which OE has a similarly wide range in thickness (Weiler \& Farbman, 1997). Weiler \& Farbman (1997) also found regional variation in thickness, noting that OE on convex structures was typically thicker than that on concave structures. However, note that here we observed thinner $\mathrm{OE}$ along the convex peripheral edges of turbinals.

The regional variation in OE thickness, which could be typical of mammals, complicates our ability to use epithelial thickness as a criterion for annotating OE limits. A bigger limitation is that the transition of OE to ciliated respiratory epithelium can be difficult to detect (Yohe, Hoffmann \& Curtis, 2018), as was the case in our study. As epithelia become thinner, they may closely approach voxel size. This means transitions must be abrupt to be accurately detected. Nonetheless, OE may be clearly detectable based on relative thickness and its greater degree of radioopacity compared to adjacent respiratory epithelium, even if its precise boundaries are not detectable. Our observations, supported by statistical results, indicate OE could be reliably identified blindly in the adult Cynopterus, but not in the fetal Desmodus. The inability to identify OE in Desmodus was likely related to the small size of the specimen and perhaps less differentiation of the OE.

\section{Identification of mucosae using histology and diceCT}

Compared to the sole use of epithelial thickness in annotation of OE on diceCT images, adding the criterion of lamina propria radioopacity yields a better match of perimeters to that of histology-informed perimeter measures. Although paired perimeter measurements suggest thickness alone was a highly effective criterion for identifying PE in the adult bat, blind annotations included some unusually thick respiratory epithelium, overestimating the amount of 
533 OE rostrally (Fig S3). The combined criteria for blind annotations produced a better match 534 rostrally (Fig. 4). OE thickness produced a very poor match in perimeter measurements in the 535 fetal bat, while the combined criteria led to identification of $\mathrm{OE}$ in precisely the same range of 536 slices as the histology-annotations, with a very close correspondence of perimeter measures (Fig. 537 4). The criteria used to assess the entire mucosa (epithelium plus lamina propria) may be suitable 538 for analysis mucosae of immature individuals comprising a cross-sectional age sample. This will 539 need to be assessed using earlier stages of prenatal animals.

540 Our study suggests glandular tissue adds to radioopacity after iodine infiltration, as does 541 covering epithelium. This was noted also by Yohe et al (2018). In this respect, it should be noted 542 that thickness of olfactory and respiratory mucosae varies greatly and can overlap in range of 543 thickness. Smith et al. (2021) related this mostly to the composition of the lamina propria.

544 Respiratory mucosa has thickened lamina propria when it is highly vascular or highly glandular.

545 In the former case, large venous sinuses may be visible (as is seen in the adult bat studied here

546 (Fig. 2c). In either instance, the epithelia of such mucosae are often thin; and because these 547 epithelia are closer to voxel dimensions they may be poorly resolved. On the other hand,

548 olfactory mucosa has a broad range of thickness with regional variation (Smith et al., 2021).

549 Bowman's glands are a reliable indicator, but the amount of glandular tissue may vary; this can

550 relate to differences in mucosa thickness, as seen in the convex versus concave sides of some 551 turbinals (e.g., see Fig. 3d). This tissue-level complexity means that an observer may be forced to 552 occasionally rely on epithelium thickness alone as a criterion for blind identification of OE.

553 However, the availability of a representative histological specimen is essential for 554 interpretations.

555 Certain limitations of the present study will require additional scrutiny. The better 556 visualized epithelia in diceCT of Cynopterus compared to Desmodus seems quite explainable 557 based on the thinner OR in the latter (very near voxel size). However, Bowman's glands were 558 not as discretely visible in our study compared to respiratory glands identified by Yohe et al. 559 using diceCT (2018, see fig. 2, therein). It is notable that Yohe et al. used about twice the 560 concentration of iodine and longer durations of staining compared to the present study.

561 Therefore, future studies should explore different durations of iodine staining for effectiveness in 562 identification of epithelia and Bowman's glands. On the other hand, Yohe et al. (2018) used 563 specimens stored in 10\% formalin, while here we examined specimens stored for decades in 564 ethanol. So another area of exploration should be the effectiveness of diceCT for identification or 565 nasal glands and epithelia in specimens stored in different fixative (see further discussion in 566 Hedrick et al., 2018).

567

568

Conclusions

Although diceCT is, as yet, only a match for light microscopy at low magnifications, our 
570

571

572

573

574

575

576

577

578

579

580

581

582

583

584

585

586

587

588

589

590

591

592

593

594

595

596

597

598

599

600

601

602

603

604

study indicates diceCT slices offer a valuable tool to annotate transitions in mucosa type within the nasal cavity. Reliance on epithelial thickness alone may suffice as an identifier of OE, particularly in the case of specimens that are well-stained, with mature, relatively thick olfactory epithelium, and given sufficient resolution. However, the use of combined criteria that interpret glandular composition of the lamina propria, along with epithelial thickness, helps to avoid false positive identification. In addition, immature specimens may exhibit characteristics of olfactory glands that can aid in identification of olfactory mucosa, even when the olfactory epithelium by itself is not completely discrete, as shown here with a fetal bat. We suggest that histology from one reference specimen of the species would be sufficient to aid in detecting epithelial transitions using diceCT.

Thus, diceCT can greatly reduce destructive methods, and at the same time greatly increase sample sizes, with less artefactual changes than occurs with histological processing. A combination of diceCT and $\mu \mathrm{CT}$ of the same specimens will allow a fuller understanding of what type(s) of mucosa line each turbinal. This would provide a firmer basis, or cautionary caveats, for the use of individual bones such as turbinals as proxies for a particular function (e.g., Van Valkenburgh et al., 2014; Martinez et al., 2018). This also has important application to future quantitative studies to further our understanding of the link between OE surface area and ecological variables (e.g., Yee et al., 2016), and in the study of fluid dynamics in the nasal airways (Craven et al., 2010; Ranslow et al., 2014).

\section{Acknowledgements}

We are grateful to Adam Hartstone-Rose, Deborah Bird, and one anonymous reviewer for numerous constructive comments that substantially improved our manuscript. We also thank Chris Vinyard for scanning one of the specimens used in this study.

\section{References}

Adams DR. 1972. Olfactory and non-olfactory epithelia in the nasal cavity of the mouse, Peromyscus. Am J Anat 133:37-50.

Bhatnagar, KP. 2008. The brain of the common vampire bat, Desmodus rotundus murinus (Wagner, 1840): a cytoarchitectural atlas. Braz. J. Biol. 68: 583-599.

Bhatnagar KP, Kallen FC. 1975. Quantitative observations on the nasal epithelia and olfactory innervation in bats. Acta Anat 91:272-282.

Buytaert J, Goyens J, De Greef D, Aerts P, Dirckx J. 2014. Volume shrinkage of bone, brain, and muscle tissue in sample preparation for micro-CT and light sheet fluorescence microscopy (LSFM). Microscopy and Microanalysis 20: 1208-1217. 
605 Chamanza R, Wright JA. 2015. A review of the comparative anatomy, histology, physiology and 606 pathology of the nasal cavity of rats, mice, dogs and non-human primates. relevance to inhalation 607 toxicology and human health risk assessment. J Comp Pathol 153:287-314.

608 Craven BA, Paterson EG, and Settles GS. 2010. The fluid dynamics of canine olfaction: unique 609 nasal airflow patterns as an explanation of macrosmia. J Roy Soc Interface 7:933-943

610 Cox PG and Jeffery NS (2011) Reviewing the morphology of the jaw-closing musculature in 611 squirrels, rats, and guinea pigs with contrast-enhanced microCT. Anat Rec 294, 915-928.

612 DeLeon VB, Smith TD. 2014. Mapping the nasal airways: using histology to enhance CT-based three-dimensional reconstruction in Nycticebus. Anat Rec 297:2113-2120.

614 Dennis JC, Aono S, Vodyanoy VJ, Morrison EE. 2015. Development, morphology, and 615 functional anatomy of the olfactory epithelium. In: Doty RL (ed), Handbook of olfaction and 616 gustation, $3^{\text {rd }}$ Ed. Wiley: New York. p 93-107.

617 Dieulafé, L. 1906. Morphology and embryology of the nasal fossae of vertebrates. Ann Otol 618 Rhinol Laryngol 15:1-584.

619 Dickinson E, Basham C, Rana A, Hartstone-Rose A. 2019. Visualization and quantification of 620 digitally dissected muscle fascicles in the masticatory muscles of Callithrix jacchus using 621 nondestructive DiceCT. Anat Rec 302:1891-1900

622 Dickinson E, Kolli S, Schwenk A, Davis CA, Hartstone-Rose A. 2020. DiceCT analysis of the 623 extreme gouging adaptations within the masticatory apparatus of the aye-aye (Daubentonia 624 madagascariensis). Anatomical Record, 303:282-294.

625 Fox CH, Johnson FB, Whiting J, Roller PP. 1985. Formaldehyde fixation. J Histochem 626 Cytochem 33, 845-853.

627 Gross EA, Swenberg JA, Fields S, Popp JA. 1982. Comparative aspects of the nasal cavity in 628 rats and mice. J Anat 135:83-88.

629 Gignac PM, Kley NJ. 2014. Iodine-enhanced micro-CT imaging: Methodological refinements 630 for the study of the soft-tissue anatomy of post-embryonic vertebrates. J. Exp. Zool. (Mol. Dev. 631 Evol.) 322B:166-176.

632 Gignac PM, Kley NJ, Clarke JA, Colbert MW, Morhardt AC, Cerio D, Cpst IN, Cox PG, Daza 633 JD, Early CM, Echol MS, Henkelman RM, Herdina AN, Holliday CM, Li Z, Mahlow K, 634 Merchant S, Müller J, Orsbon CP, Paluh DJ, Thies ML, Tsai HP, Witmer LM. (2016). Diffusible 635 iodine-based contrast-enhanced computed tomography (diceCT): an emerging tool for rapid, 636 high-resolution, 3-D imaging of metazoan soft tissues. J. Anat. 228, 889-909.

637 Girard R, Zeineddine HA, Orsbon C, Tan H, Moore T, Hobson N, Shenkar R, Lightle R, Shi C, 638 Fam MD, Cao Y, Shen L, Neander AI, Rorrer A, Gallione C, Tang AT, Kahn ML, Marchuk DA, 639 Luo Z, Awad IA. 2016. Micro-computed tomography in murine models of cerebral cavernous 640 malformations as a paradigm for brain disease. J Neurosci Methods 271, 14-24. 
641 Harkema JR, Carey SA, Pestka JJ. 2006. The nose revisited: a brief review of the comparative 642 structure, function, and toxicologic pathology of the nasal epithelium. Toxicol Pathol 34:252643269.

644 Hedrick BP, Yohe L, Vander Linden A, Dávalos LM, Sears K, Sadier A, Rossiter SJ, Davies, 645 KTJ, Dumon, E. 2018. Assessing soft-tissue shrinkage estimates in museum specimens imaged 646 with diffusible iodine-based contrast-enhanced computed tomography (diceCT). Microscopy and 647 Microanalysis doi: 10.1017/S1431927618000399

648 Leonard KC, Worden N, Boettcher ML, Dickinson E, Hartsone-Rose A. 2021. Effects of long649 term ethanol storage on muscle architecture. Anatomical Record https://doi.org/10.1002/ar.24638

650 Maier W, Ruf I. 2014. Morphology of the nasal capsule of primates - with special reference to 651 Daubentonia and Homo. Anatomical Record, 297: 1985-2006.

652 Martinez Q, Lebrun R, Achmadi AS, Esselstyn JA, Evans AR, Heaney LR, Miguez RP, Rowe 653 KC, Fabre P-H. 2018. Convergent evolution of an extreme dietary specialization, the olfactory 654 system of worm-eating rodents. Scientific Reports 8:17806 | DOI:10.1038/s41598-018-35827-0

655 Orsbon CP, Gidnark NJ, Ross CF. 2018. Dynamic Musculoskeletal Functional Morphology: 656 Integrating diceCT and XROMM. Anatomical Record, 301: 378-406.

657 Pang B, Yee KK, Lischka FW, Rawson NE, Haskins, ME, Wysocki CJ, Craven BA, Van 658 Valkenburgh B. 2014. The influence of nasal airflow on respiratory and olfactory epithelial 659 distribution in felids. J. Exp. Biol. 219,1866-1874.

660 Ranslow AN, Richter JP, Neuberger T, Van Valkenburgh B, Rumple CR, Quigley AP, Pang B, 661 Krane MH, Craven BA. 2014. Reconstruction and morphometric analysis of the nasal airway of 662 the white-tailed deer (Odocoileus virginianus) and implications regarding respiratory and 663 olfactory airflow. Anat Rec 297: 2138-2147.

664 Reinholt LE, Burrows AM, Eiting TP, Dumont ER, Smith TD. 2009. Brief communication: 665 Histology and microCT as methods for assessing facial suture patency. American Journal of 666 Physical Anthropology, 138:499-506.

667 Rolls G, Farmer N. 2008. Artifacts in histological and cytological preparations. Leica 668 Biosystems: Wetzlar, Germany.

669 Santana SE. 2018. Comparative anatomy of bat jaw musculature via diffusible iodine-based 670 contrast-enhanced computed tomography. Anatomical Record 301:267-278

671 Smith TD, Bhatnagar KP, Rossie JP, Docherty BA, Burrows AM, Mooney MP, Siegel MI. 2007. 672 Scaling of the first ethmoturbinal in nocturnal strepsirrhines: olfactory and respiratory surfaces.

673 Anat Rec 290:215-237.

674 Smith TD, Bhatnagar KP. 2019. Anatomy of the olfactory system. In: Doty R Ed). Handbook of 675 Clinical Neurology Elsevier, Vol. 164 ( $3^{\text {rd }}$ series) Smell and Taste. New York: Elsevier. pp 1767628. 
677 Smith TD, DeLeon VB, Vinyard CJ, Young JW. 2020. Skeletal anatomy of the newborn primate. 678 Cambridge: Cambridge University Press.

679 Smith TD, BA Craven, SM, Engel, B Van Valkenburgh, VB DeLeon. 2021. "Mucosal maps" of 680 the canine nasal cavity: micro-computed tomography and histology. Anatomical Record, 304:

$681 \quad 127-138$

682 Tahara R and Larsson HCE (2013) Quantitative analysis of microscopic x-ray computed 683 tomography imaging: Japanese quail embryonic soft tissues with iodine staining. J Anat 223, $684297-310$.

685 Weiler E, Farbman AI. 1997. Proliferation in the Rat Olfactory Epithelium: Age-Dependent Changes. J 686 Neurosci 17:3610-3622.

687 Van Valkenburgh B, Pang B, Bird D, Curtis C, Yee K, Wysocki C, Craven B. 2014. Respiratory 688 and olfactory turbinals in feliform and caniform carnivorans: the influence of snout length. Anat 689 Rec 297:2065-2079.

690 Vickerton P, Jarvis J, Jeffery N 2013. Concentration-dependent specimen shrinkage in iodine691 enhanced microCT. J Anat 223:185-193.

692 Yapuncich GS, Kemp AD, Griffith DM, Gladman JT, Ehmke E, Boyer DM. 2019. A digital 693 collection of rare and endangered lemurs and other primates from the Duke Lemur Center. PLOS 694 ONE 14(11): e0219411. https://doi.org/10.1371/journal.pone.0219411

695 Yohe LR, Hoffmann S, Curtis A. 2018 Vomeronasal and olfactory structures in bats revealed by 696 diceCT clarify genetic evidence of function. Front Neuroanat 12:32.

697 Yee KK, Craven BA, Wysocki C J, Van Valkenburgh, B. 2016. Comparative morphology and 698 histology of the nasal fossa in four mammals: gray squirrel, bobcat, coyote, and white-tailed 699 deer. The Anatomical Record 299:840-852. 
700

701

702

703

704

705

706

707

708

709

710

711

712

713

714

715

716

717

718

719

720

721

722

723

724

725

726

727

728

729

730

731

732

733

734

735

736

737

738

\section{FIGURE LEGENDS}

Figure 1: Matching cross-sectional levels in ethmoturbinal region of the fetal Desmodus (top row) and adult Cynopterus (bottom row). Spatially dispersed structures viewed in a histological section of the fetal bat (a), such as the eye (E), first ethmoturbinal (ET I), septal cartilage (SC), maxilla (Mx), frontal bone (Fr), and or lateral recess (LR) can be seen in the diceCT scan (b) or $\mu \mathrm{CT}$ (c) slices. Note soft tissue structures of diceCT and osseous structures of $\mu \mathrm{CT}$ have been carefully aligned to histology. In the adult bat (d), histology reveals mucosa and supporting bones of the turbinals. Note the mucosal contours of ET I, an epiturbinal (epT) and the maxilloturbinal (MT) of the diceCT slice (e) are in alignment with histology. Similarly, the bones of these turbinals seen in the $\mu \mathrm{CT}$ slices are well aligned with histology. Also, note the epT contour is clearly visible in the $\mu \mathrm{CT}$ slice, and thus was compared to diceCT and histology to assess shrinkage. $\mathrm{CP}$, cribriform plates; $\mathrm{NpD}$, nasopharyngeal duct; vET II, ventral accessory lamella of ethmoturbinal II. Scale bars: a-c, $1 \mathrm{~mm}$; d, $0.5 \mathrm{~mm}$; e,f, $250 \mu \mathrm{m}$.

Figure 2: Paired measurements of identical structures in adult Cynopterus showing corresponding, aligned $\mu$-CT slices (a,d), diceCT slices (b, e) and histological sections (c, f). a-c) Cross-sections through a caudal portion of the maxilloturbinal (MT) at a matching level. Note the MT is more robust in the $\mu$-CT slice (a) compared to the other images; the slightly thinner mucosa in diceCT (b) and histology (c) may correspond to shrinkage of the lamina propria, which contains mostly venous sinuses (*). d-f) Cross-sections through the snout of Cynopterus revealing freely projecting epiturbinals (epT), an accessory projection of the first ethmoturbinal (ET I). A roughly similar contour is visible using all three imaging techniques. Note the respiratory epithelium is lined by a thin (scale $=20 \mu \mathrm{m})$ pseudostratified, ciliated epithelium (inset, c; arrowhead indicate cilia). Respiratory gland (RG) masses are seen near the root of the $\mathrm{MT}$, and are isolated radioopaque masses in diceCT slices (b). The epT contains a dense lamina propria dorsally (f). A magnified view of the olfactory mucosa $(\mathrm{OM})$ reveals the lamina propria (LP) is dense with Bowman's glands (BG), and the epithelium (Ep) is far thicker than that lining the MT (inset, f; scale $=20 \mu \mathrm{m}$ ). g, h) Paired measurements of the MT and epT at matching levels, revealing that $\mu$-CT measurements are larger compared to diceCT or (especially) histology at almost all matching levels. D, gland duct. Scale bars: a, b, d, e, $250 \mu \mathrm{m}$; c, $0.5 \mathrm{~mm}$; f, $100 \mu \mathrm{m}$, insets, $20 \mu \mathrm{m}$.

Figure 3: Matching histology and diceCT of Desmodus rotundus revealing characteristics of olfactory mucosa that are apparent in diceCT. a) The first ethmoturbinal (ET I) shown in a Gomori-trichrome stained section at its point of attachment to the nasal fossa "roof." On its medial face is a thick olfactory mucosa (OM). b) An enlarged view of the OM. OM thickness corresponds in part to an olfactory epithelium in which the bodies of sensory neurons are staggered throughout its depth; note rows of nuclei (OSN). A greater extent of its thickness corresponds to the lamina propria, which is home to numerous Bowman's glands (BG) and olfactory nerves $(\mathrm{ON})$. c) The same turbinal shown in an aligned diceCT slice. The entire mucosal depth of ET I is radiopaque on its medial side. The septum that faces ET I has a thinner 
739

740

741

742

743

744

745

746

747

748

749

750

751

752

753

754

755

756

757

758

759

760

761

762

763

764

765

766

767

768

769

770

771

772

773

774

775

776

777

mucosa. This mucosa (enlarged in inset, space between bars) has a greater radioopacity than the septal cartilage (S) that supports it. d) A ventral accessory lamella of ethmoturbinal II (vET II) lined with olfactory OM. e) An enlargement of the free margin of this turbinal revealing a thick olfactory epithelium (OE) and densely glandular lamina propria. f) An aligned diceCT slice of ET II, showing the turbinal is almost completely radioopaque. Note, however, small patches of non-OE are easily identifiable using histology (d, black arrowhead), but are less distinct in diceCT (f, white arrowhead). Also note, in many locations the thickness of the olfactory epithelium in the fetus closely approaches the voxel dimension of $\sim 18 \mu \mathrm{m}$ (b). In contrast, the olfactory epithelium in Cynopterus more greatly exceeds the voxel dimension of $\sim 21 \mu \mathrm{m}(\mathrm{e})$. Scale bars: a, $100 \mu \mathrm{m}$; b, $20 \mu \mathrm{m}$; c, $250 \mu \mathrm{m}$; d, $200 \mu \mathrm{m}$; e, $100 \mu \mathrm{m}$; f, $0.5 \mathrm{~mm}$.

Figure 4: Perimeter measurements of olfactory epithelia in the two bats. a, c) Ethmoturbinal I (ET I); b, d) septum and roof. Measurements of matching levels are plotted for slices annotated according to histological observations (blue symbols) and those annotated blindly based on mucosal appearance in diceCT.

Supplemental Figure 1: DiceCT slices of Cynopterus sphinx, showing sites measured for epithelial thickness (numbers indicate slice numbers; higher numbers are more rostral). Measurements were made on histological sections in the same region as this slice series. Site a: ridge or apex of ET I, which orients superiorly at rostral extent (1614) and then leans medially as ET I is followed caudally. Site b: septal epithelium found adjacent to site a. Site c,inferior-most limit of medial lamina of ET I. Site d: septal epithelium found adjacent to site d. Site e, medial side of the frontoburbinal. Site f: superior-most tip of the nasoturbinal. Site g: "roof" of nasal cavity, opposite site $\mathrm{f}$. Locations $\mathrm{c}$ and $\mathrm{d}$ were selected as ventral sites where respiratory epithelium is expected.

Supplemental Figure 2: diceCT (a-d) and histological views (f-i) of the nasal cavity in adult Cynopterus, revealing the hazards of using thickness alone as a criterion for inferring epithelial type.. After aligning CT to histology, the diceCT slices were assessed for changes in nasal epithelial thickness. a) In the region of the rostral projection of the first ethmoturbinal (ET I), noticeably thicker epithelium is observed on the superior side of ET I, and on adjacent surfaces of the nasal septum (NS) and "roof" of the nasal cavity (b). Arrows indicate transitions from thicker (presumably olfactory) to thinner (presumably respiratory) epithelia. An observer who was blind to the histology marked where the change in thickness was evident using ImageJ software (c). d) Rostral to ET I, thicker epithelium exists on the roof of the nasal cavity (borders annotated). e) approximate levels of slices in plate a and $d$ are indicated by green dashed lines on the graph of septal OE. f, g) Low and higher magnification views of the histology section matching plate a. Note a transition in epithelial thickness can be detected at low magnification (arrows). The thicker epithelium has numerous rows of nuclei of olfactory sensory neurons (OSN), and the transition to thinner non-OE is nearly abrupt, with a shift to respiratory epithelium (arrowheads indicate cilia). h, i) Low and higher magnification of histology matching the slice in plate $\mathrm{d}$. Although this is indeed a thick epithelium, cilia can be seen by light 
778 microscopy (arrowheads) indicating it is a respiratory type. BG, Bowman's glands; MT,

779 maxilloturbinal. Scale bars, a-d, $0.5 \mathrm{~mm}$; f, h, $150 \mu \mathrm{m}$; g, I, $10 \mu \mathrm{m}$.

780 Supplemental Figure 3: Olfactory epithelial (OE) perimeter on the first ethmoturbinals (ET I)

781 and the nasal septum as measured based on diceCT slices annotated blindly, based on trial one of

782 the third analysis: epithelial thickness ("blind") and diceCT annotated based on histology

783 ("histology"). a) OE as assessed by the two methods was close for most of the length of ET I, but

784 the methods diverged anteriorly (right side of the graph). A similar phenomena was observed for

785 septal measurements (b).

786

787 


\section{Figure 1}

Figure 1 showing alignment of histology to CT

Figure 1: Matching cross-sectional levels in ethmoturbinal region of the fetal Desmodus (top row) and adult Cynopterus (bottom row). Spatially dispersed structures viewed in a histological section of the fetal bat (a), such as the eye (E), first ethmoturbinal (ET I), septal cartilage $(\mathrm{SC})$, maxilla $(\mathrm{Mx})$, frontal bone $(\mathrm{Fr})$, and or lateral recess $(\mathrm{LR})$ can be seen in the diceCT scan (b) or $\mu \mathrm{CT}$ (c) slices. Note soft tissue structures of diceCT and osseous structures of $\mu \mathrm{CT}$ have been carefully aligned to histology. In the adult bat $(d)$, histology reveals mucosa and supporting bones of the turbinals. Note the mucosal contours of ET I, an epiturbinal (epT) and the maxilloturbinal (MT) of the diceCT slice (e) are in alignment with histology. Similarly, the bones of these turbinals seen in the $\mu \mathrm{CT}$ slices are well aligned with histology. Also, note the epT contour is clearly visible in the $\mu \mathrm{CT}$ slice, and thus was compared to diceCT and histology to assess shrinkage. CP, cribriform plates; NpD, nasopharyngeal duct; vET II, ventral accessory lamella of ethmoturbinal II. Scale bars: a-c, $1 \mathrm{~mm}$; d, $0.5 \mathrm{~mm}$; e,f, $250 \mu \mathrm{m}$. 


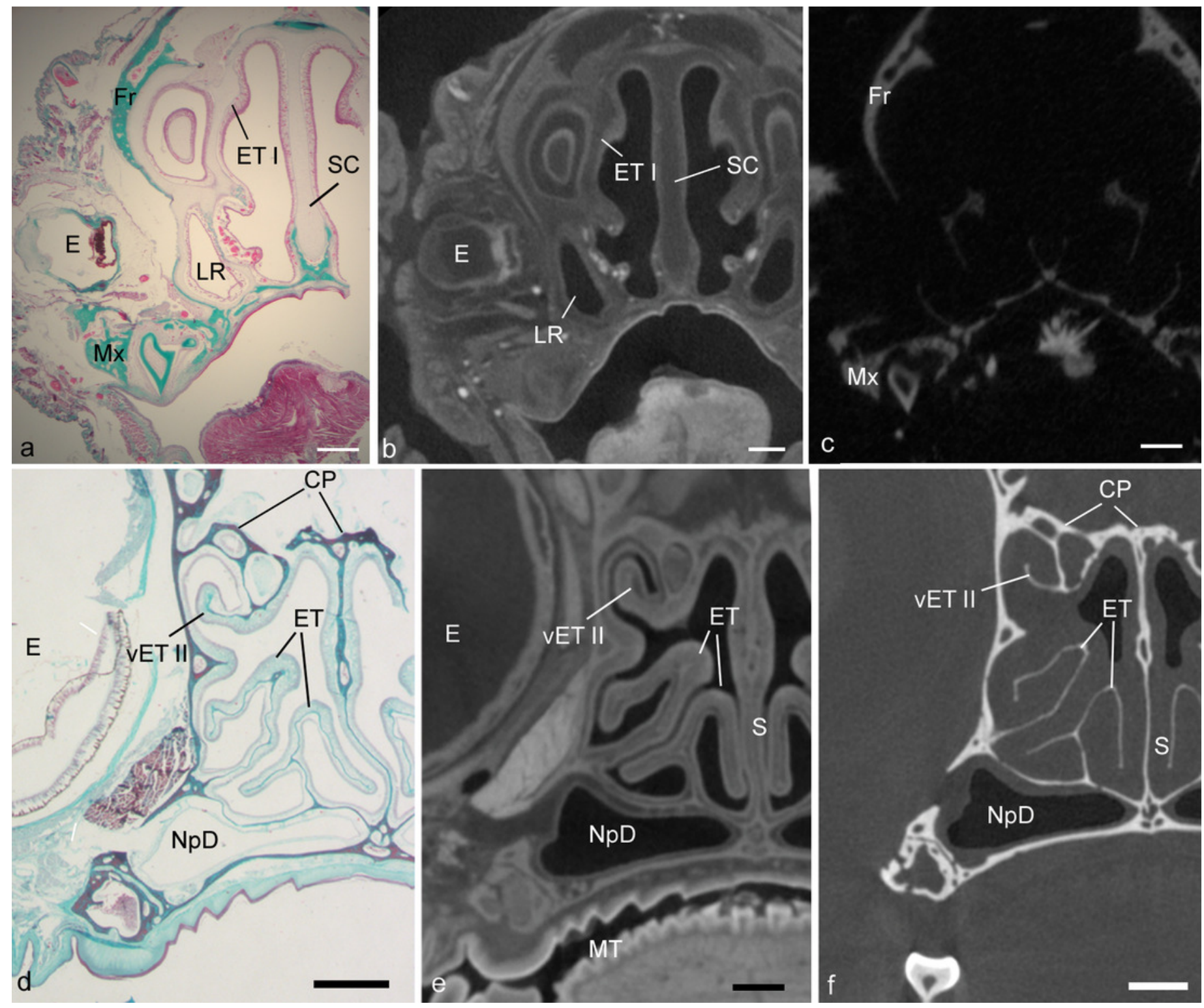




\section{Figure 2}

Figure 2 showing matching histology, diceCT, and microCT

Figure 2: Paired measurements of identical structures in adult Cynopterus showing corresponding, aligned $\mu$-CT slices $(a, d)$, diceCT slices (b, e) and histological sections (c, f). ac) Cross-sections through a caudal portion of the maxilloturbinal (MT) at a matching level. Note the MT is more robust in the $\mu$-CT slice (a) compared to the other images; the slightly thinner mucosa in diceCT (b) and histology (c) may correspond to shrinkage of the lamina propria, which contains mostly venous sinuses $(*)$. d-f) Cross-sections through the snout of Cynopterus revealing freely projecting epiturbinals (epT), an accessory projection of the first ethmoturbinal (ET I). A roughly similar contour is visible using all three imaging techniques. Note the respiratory epithelium is lined by a thin (scale $=20 \mu \mathrm{m}$ ) pseudostratified, ciliated epithelium (inset, c; arrowhead indicate cilia). Respiratory gland (RG) masses are seen near the root of the MT, and are isolated radioopaque masses in diceCT slices (b). The epT contains a dense lamina propria dorsally (f). A magnified view of the olfactory mucosa (OM) reveals the lamina propria (LP) is dense with Bowman's glands (BG), and the epithelium (Ep) is far thicker than that lining the MT (inset, f; scale $=20 \mu \mathrm{m}$ ). $\mathrm{g}, \mathrm{h}$ ) Paired measurements of the MT and epT at matching levels, revealing that $\mu$-CT measurements are larger compared to diceCT or (especially) histology at almost all matching levels. D, gland duct. Scale bars: a, b, d, e, $250 \mu \mathrm{m} ; \mathrm{c}, 0.5 \mathrm{~mm}$; f, $100 \mu \mathrm{m}$, insets, $20 \mu \mathrm{m}$. 

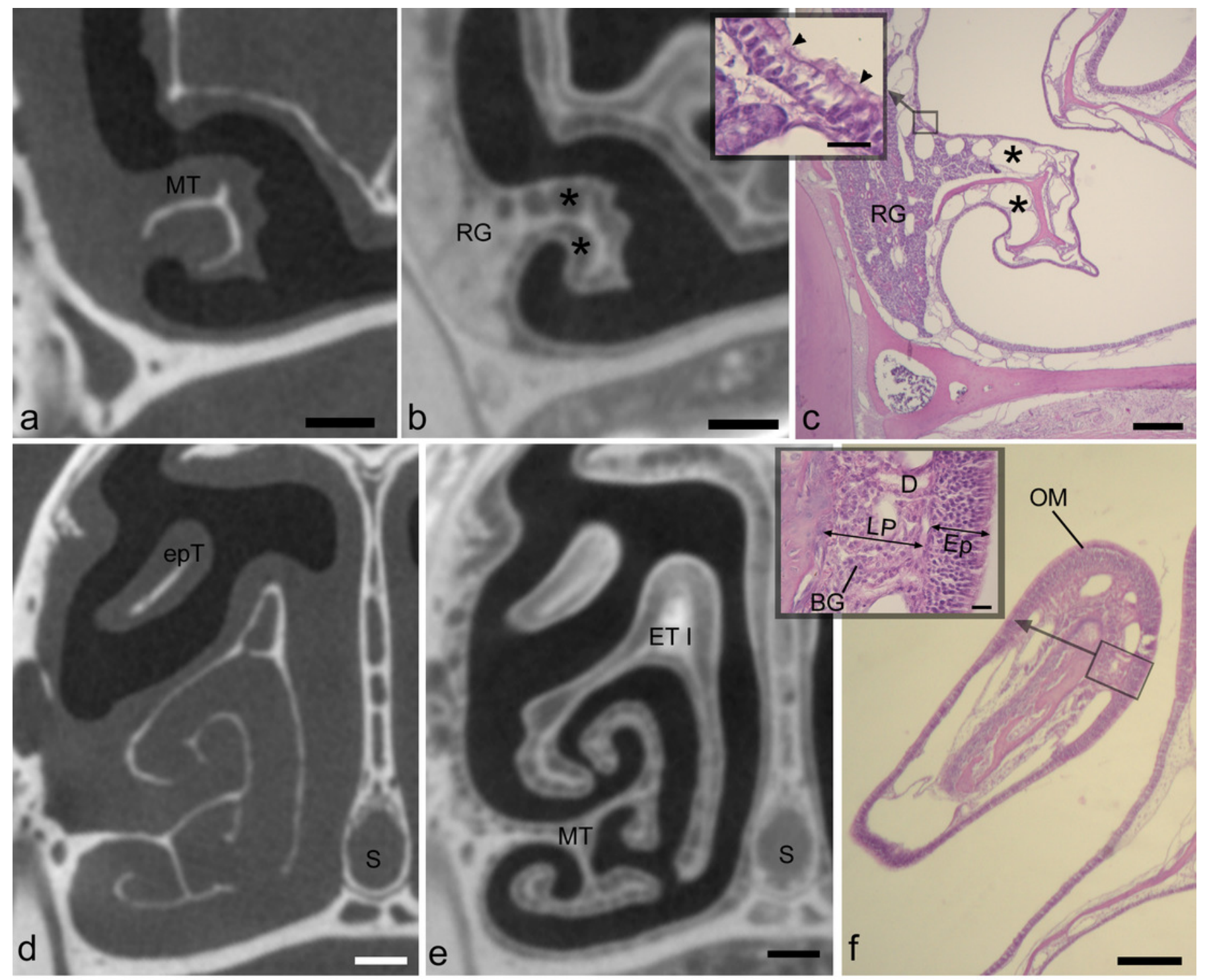

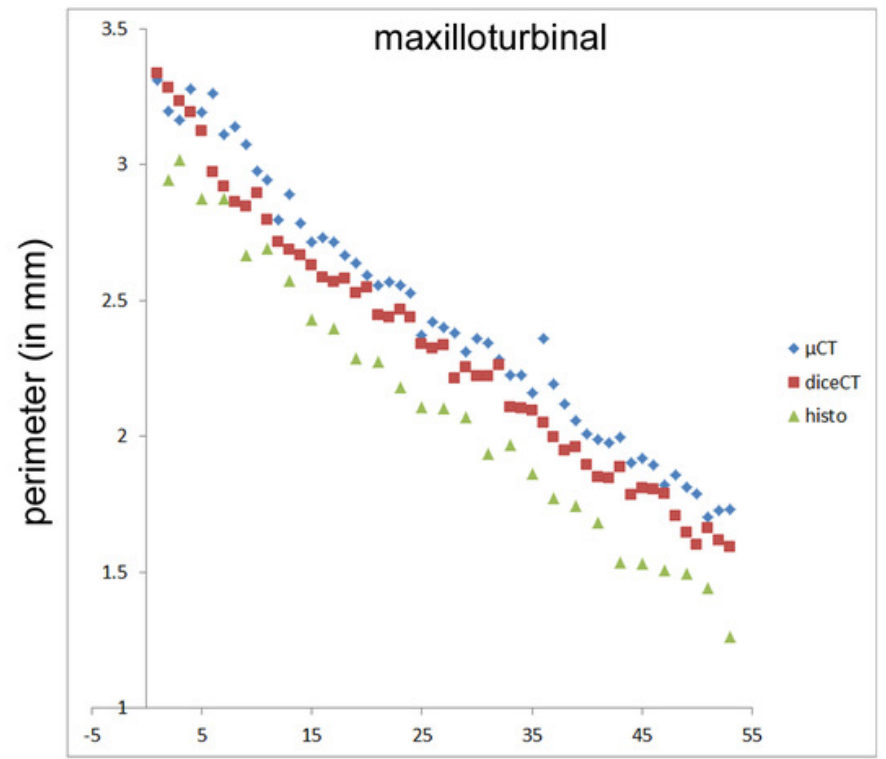

g

slice levels (rostral to caudal)

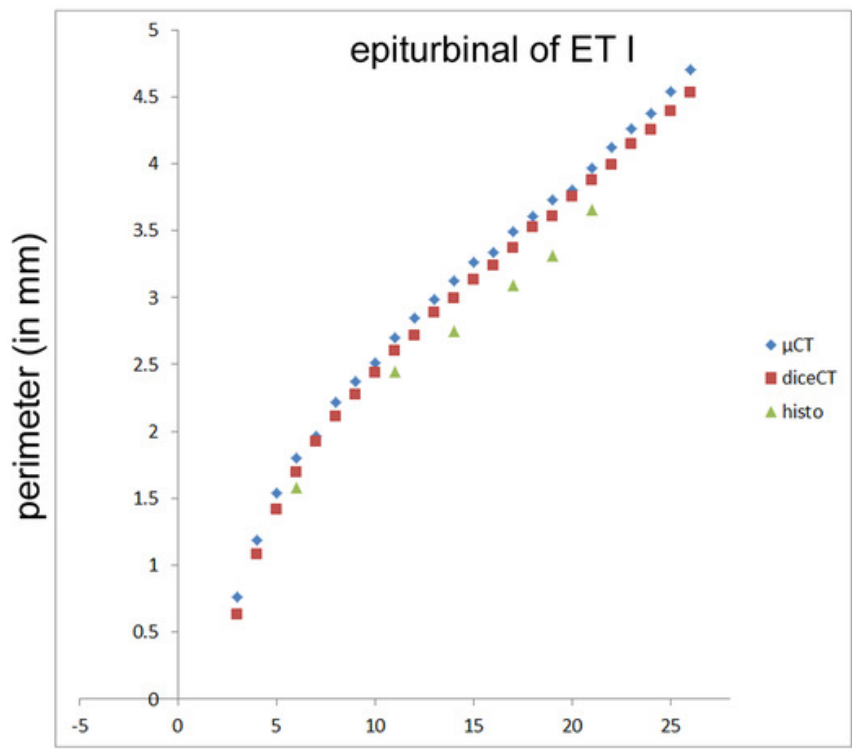

h slice levels (rostral to caudal) 


\section{Figure 3}

Figure 3, showing diceCT aspects indicating mucosal identity

Figure 3: Matching histology and diceCT of Desmodus rotundus revealing characteristics of olfactory mucosa that are apparent in diceCT. a) The first ethmoturbinal (ET I) shown in a Gomori-trichrome stained section at its point of attachment to the nasal fossa "roof." On its medial face is a thick olfactory mucosa (OM). b) An enlarged view of the OM. OM thickness corresponds in part to an olfactory epithelium in which the bodies of sensory neurons are staggered throughout its depth; note rows of nuclei (OSN). A greater extent of its thickness corresponds to the lamina propria, which is home to numerous Bowman's glands (BG) and olfactory nerves (ON). c) The same turbinal shown in an aligned diceCT slice. The entire mucosal depth of ET I is radiopaque on its medial side. The septum that faces ET I has a thinner mucosa. This mucosa (enlarged in inset, space between bars) has a greater radioopacity than the septal cartilage (S) that supports it. d) A ventral accessory lamella of ethmoturbinal II (VET II) lined with olfactory OM. e) An enlargement of the free margin of this turbinal revealing a thick olfactory epithelium (OE) and densely glandular lamina propria. f) An aligned diceCT slice of ET II, showing the turbinal is almost completely radioopaque. Note, however, small patches of non-OE are easily identifiable using histology (d, black arrowhead), but are less distinct in diceCT (f, white arrowhead). Also note, in many locations the thickness of the olfactory epithelium in the fetus closely approaches the voxel dimension of $\sim 18 \mu \mathrm{m}$ (b). In contrast, the olfactory epithelium in Cynopterus more greatly exceeds the voxel dimension of $\sim 21 \mu \mathrm{m}$ (e). Scale bars: a, $100 \mu \mathrm{m} ; \mathrm{b}, 20 \mu \mathrm{m} ; \mathrm{c}, 250 \mu \mathrm{m}$; d, $200 \mu \mathrm{m} ; \mathrm{e}, 100 \mu \mathrm{m}$; f, $0.5 \mathrm{~mm}$. 


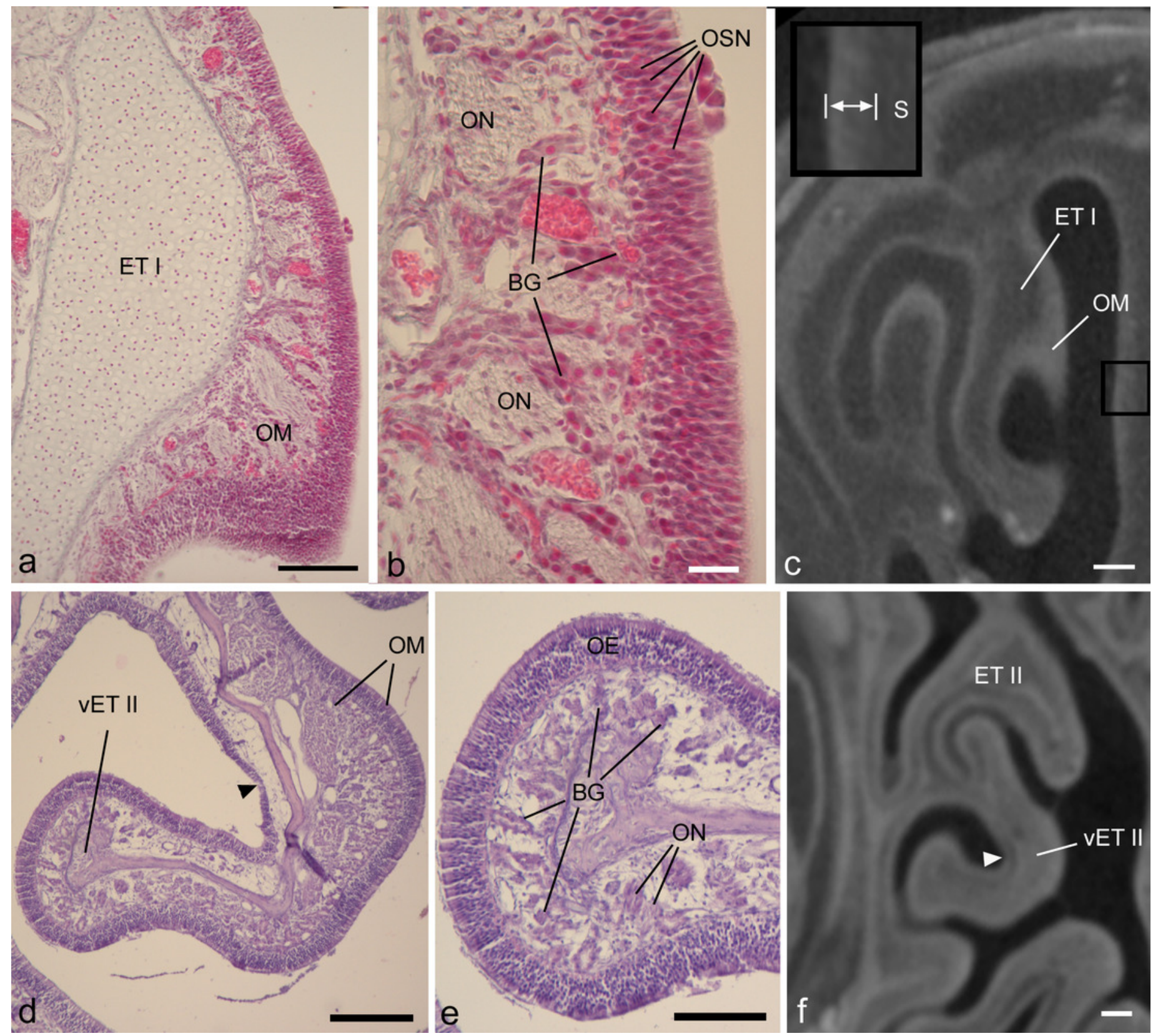




\section{Figure 4}

Graphs of blind versus histology-informed annotations

Figure 4: Perimeter measurements of olfactory epithelia in the two bats. a, c) Ethmoturbinal I (ET I); b, d) septum and roof. Measurements of matching levels are plotted for slices annotated according to histological observations (blue symbols) and those annotated blindly based on mucosal appearance in diceCT. 


\section{Cynopterus sphinx, adult}
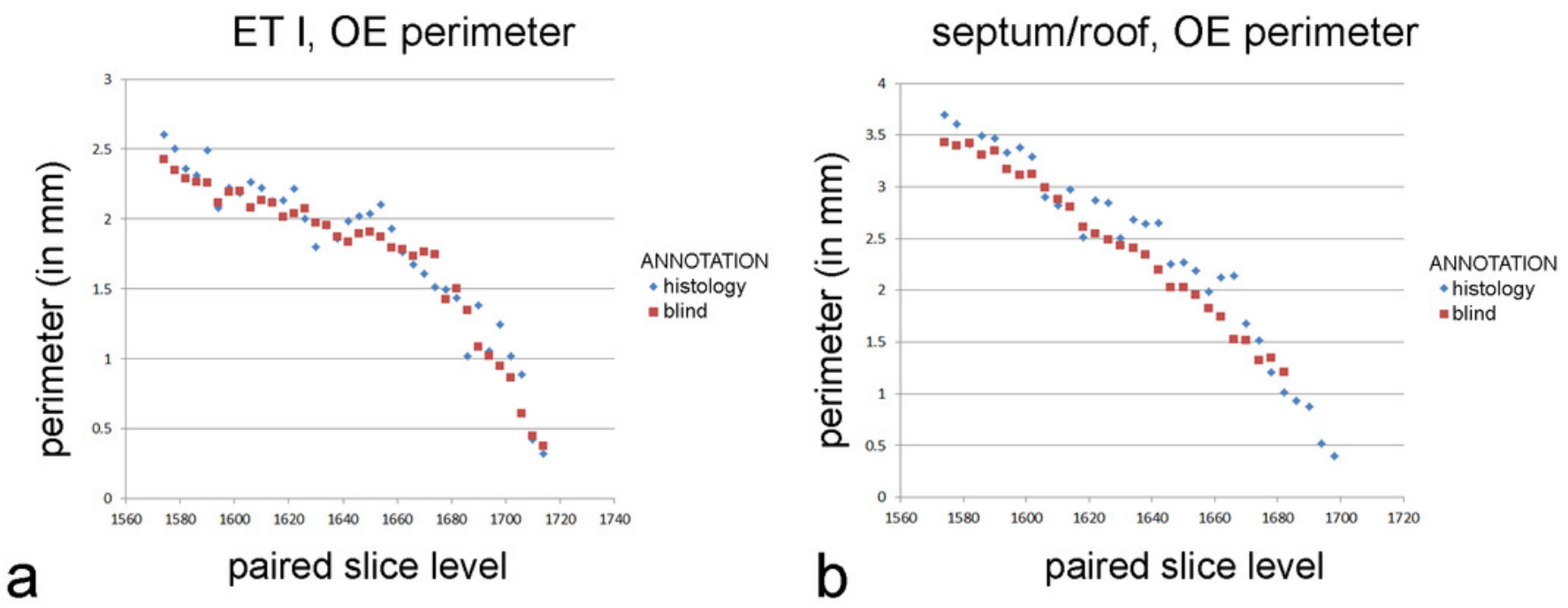

\section{Desmodus rotundus, fetal}

ET I, OE perimeter

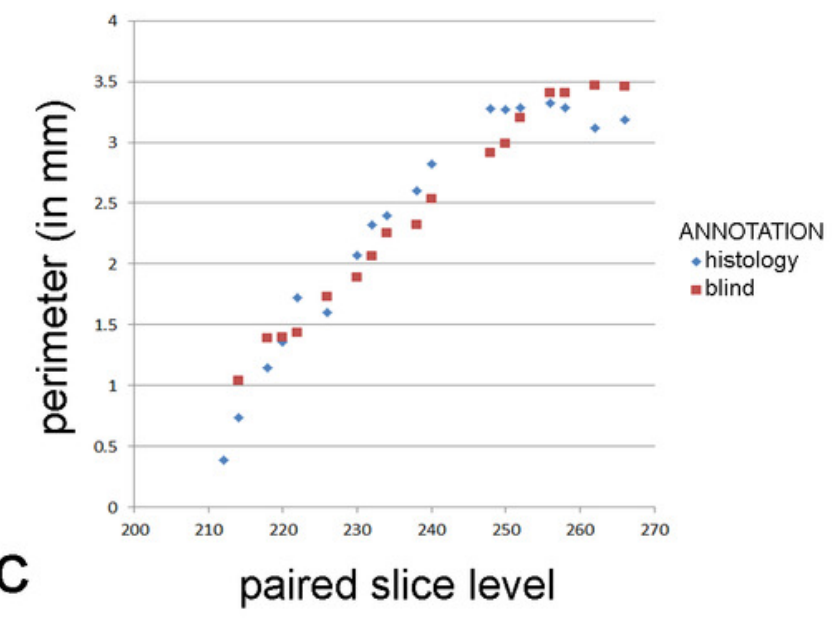

septum/roof, OE perimeter

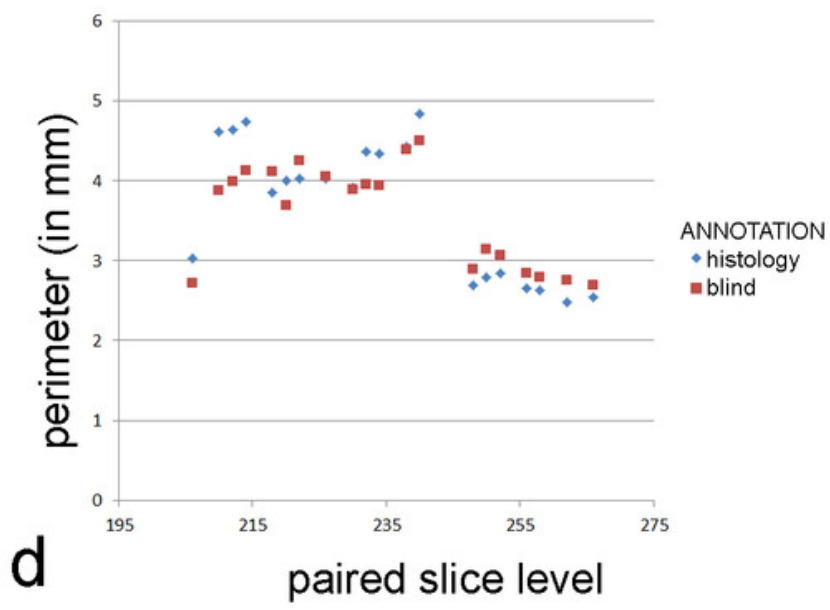




\section{Table $\mathbf{1}$ (on next page)}

Descriptive statistics of thickness (in $\mu \mathrm{m}$ ) of olfactory epithelia by site and results of single factor ANOVA 
Table 1: Descriptive statistics of thickness (in $\mu \mathrm{m}$ ) of olfactory epithelia by site and results of single factor ANOVA

SUMMARY

\begin{tabular}{lccrr}
\hline \multicolumn{1}{c}{ Groups } & Count & Sum & Average & Variance \\
\hline ETI & 24 & 1011.75 & $42.156^{1}$ & 29.505 \\
nasal septum & 23 & 1152.95 & 50.128 & 15.544 \\
FT & 9 & 453.75 & 50.417 & 31.676 \\
NT & 15 & 664.13 & $44.275^{1}$ & 55.098 \\
"roof" & 14 & 813.67 & 58.119 & 32.733 \\
\hline
\end{tabular}

ANOVA

\begin{tabular}{lcccccc}
\hline \multicolumn{1}{c}{ Source of Variation } & $S S$ & $d f$ & $M S$ & $F$ & P-value & F crit \\
\hline Between Groups & 2614.917 & 4 & 653.7291 & 21.16572 & $6.22 \mathrm{E}-12$ & 2.485885 \\
Within Groups & 2470.898 & 80 & 30.88622 & & & \\
& & & & & & \\
Total & 5085.814 & 84 & & & & \\
\hline
\end{tabular}

${ }^{1}$ LSD comparisons reveal epithelia of ET I and NT are significantly thinner compared to all other olfactory sites, but not significantly different from each other. 


\section{Table 2 (on next page)}

Results of t-tests comparing thickness (in $\mu \mathrm{m}$ ) of olfactory epithelium (OE) versus nonolfactory epithelium (non-OE) on ethmoturbinal I and the nasal septum 
1

2 TABLE 2: Results of t-tests comparing thickness (in $\mu \mathrm{m}$ ) of olfactory epithelium (OE)

3 versus non-olfactory epithelium (non-OE) on ethmoturbinal I and the nasal septum

4 comparison mean variance $t$ statistic $p$ value

5 ethmoturbinal I: $\quad$ OE $\quad 42.16 \quad 29.51 \quad 24.85 \quad \mathrm{p}<0.001$

non-OE

10.89

7.43

7 nasal septum:

$\mathrm{OE}$

50.13

16.08

32.41

$\mathrm{p}<0.001$

8

non-OE

16.08

8.99

9

10

11 